ISSN 0103-5150

Fisioter. Mov., Curitiba, v. 26, n. 1, p. 219-228, jan./mar. 2013

Licenciado sob uma Licença Creative Commons

\title{
Teste de caminhada de seis minutos em pediatria: relação entre desempenho e parâmetros antropométricos
}

\author{
Six-minute walk test in pediatrics: the relationship between \\ performance and anthropometric parameters
}

\section{Renata Tiemi Okuro $^{[a]}$, Camila Isabel Santos Schivinski ${ }^{\text {b] }}$}

[a] Doutoranda em Ciências biológicas (Fisiologia) pela Universidade Federal do Rio de Janeiro (UFRJ), mestre em Ciências pela Universidade Estadual de Campinas (Unicamp), Campinas - SP, Brasil, e-mail: re_okuro@yahoo.com.br

[b] Doutora em Saúde da Criança e do Adolescente pela Universidade Estadual de Campinas (Unicamp), professora efetiva dos cursos de Graduação e Pós-Graduação em Fisioterapia da Universidade do Estado de Santa Catarina (Udesc),

Florianópolis, SC - Brasil, e-mail: cacais@yahoo.com.br

\section{Resumo}

Introdução: Diferenças antropométricas podem influenciar o desempenho no teste de caminhada de seis minutos $\left(\mathrm{TC}_{6}\right)$. Objetivo: Analisar publicações referentes ao $\mathrm{TC}_{6}$ realizado em crianças e adolescentes e identificar a influência de parâmetros antropométricos no desempenho do teste. Materiais e métodos: Pesquisa nas bases de dados eletrônicas SciELO e MEDLINE, utilizando as palavras-chave: six-minute walk test, children, adolescents, anthropometric measures/variables, growth e exercise tolerance, na busca de estudos envolvendo $\mathrm{TC}_{6}$ em crianças e adolescentes, que apresentem correlação entre dados antropométricos e variáveis do teste. Foram identificados 45 trabalhos relacionados à busca e, após análise dos títulos, resumos e artigos na íntegra, foram incluídos 13 estudos compatíveis com o tema proposto. Resultados: 0 material elencado aborda o $\mathrm{TC}_{6}$ e sua aplicação em diferentes situações: análise de reprodutibilidade, correlação com teste cardiopulmonar máximo, indicação em diferentes doenças, valores de referência em saudáveis, parâmetro de efeito de programas de prevenção/reabilitação. Muitos trabalhos relacionam a distância percorrida no $\mathrm{TC}_{6}$ com idade e sexo, bem como com variáveis antropométricas, evidenciando frequente correlação com altura, além de idade, peso e índice de massa corporal. Outros dados como comprimento e força de membros inferiores, porcentagem de massas gorda/magra e força muscular respiratória, 
relacionados às mudanças do período de desenvolvimento infanto-juvenil, também parecem influenciar no desempenho do teste. Conclusão: Estudos indicam forte influência entre variáveis antropométricas e distância percorrida no $\mathrm{TC}_{6}$ em crianças e adolescentes, e a necessidade de que se esclareça essa associação para que não haja comprometimento do desfecho de estudos e de terapêuticas.

Palavras-chave: Teste de esforço. Criança. Antropometria. Pediatria.

\begin{abstract}
Introduction: Anthropometric differences may influence the performance in the 6-minute walk test. Objective: To analyze publications concerning the 6MWT performed in children and adolescents and to identify the influence of anthropometric parameters on test performance. Materials and methods: research in the electronic databases Medline and Scielo using to the keywords: six-minute walk test, children, adolescents, anthropometric measures/ variables, growth and exercise tolerance, in the search of studies involving 6MWT in children and adolescents, that present correlation between anthropometric data and test variables. It was identified 45 studies related to the search and after examination of the titles, abstracts and full articles, it were included 13 compatible studies with the proposed theme. Results: the selected material approach the 6MWT and your application in different situations: analysis of reproducibility, correlation with maximal cardiopulmonary exercise testing, indications in differents diseases, reference values in healthy, effect parameter of prevention/rehabilitation program. Many studies relate the covered distance in 6MWT with age and sex, as well as with anthropometric variables, showing frequent correlation with height, beyond the age, weight and body mass index. Other data such as length and strength of lower limbs, percentage of fat mass/lean and respiratory muscle strength, related to changes in the period of juvenile development, also seem to influence the test performance. Conclusion: Studies indicate a strong influence between the anthropometric variables and the covered distance in 6MWT in children and adolescents, and the need for better explanation of this association so there is no impairment of outcome studies and therapeutics.
\end{abstract}

Keywords: Exercise test. Child. Anthropometry. Pediatrics.

\section{Introdução}

0 Teste de Caminhada de seis minutos $\left(\mathrm{TC}_{6}\right)$ foi elaborado para ser um teste de caminhada prático capaz de estimar a capacidade funcional submáxima dos pacientes, através da medida da distância percorrida (DP) em um corredor plano por um período de seis minutos (1). É um teste seguro, fácil de ser administrado, reprodutível, validado e bem tolerado, sendo o que melhor se relaciona com o desempenho do paciente nas atividades de vida diária em relação a outros testes (2). Tem sido uma alternativa potencial para substituir testes de exercício máximo que, apesar de considerados padrão ouro para estimar a tolerância ao exercício, muitas vezes são de difícil execução, demandam equipamentos de alto custo e recrutamento de pessoal especializado (3).

$0 \mathrm{TC}_{6}$ avalia as respostas ao exercício de forma integrada e global por parte de todos os sistemas envolvidos na atividade física (circulação periférica e sistêmica, função hemodinâmica, unidade neuromuscular, metabolismo muscular, aparelho cardiovascular). Não oferece, porém, informações específicas sobre cada sistema, mas é um teste sensível e atualmente é o teste de caminhada mais utilizado (4). Sua aplicação em ensaios clínicos vem se expandindo, principalmente em condições cardiorrespiratórias crônicas, quando a capacidade de exercício e o nível de atividade são limitados. É indicado para avaliação da capacidade funcional, propostas epidemiológicas para pesquisas, monitorização da eficácia de intervenções, especialmente como parâmetro de resposta a programas de reabilitação pulmonar e seguimentos de protocolos fisioterapêuticos $(4,5)$. Também têm sua relevância no estabelecimento de prognóstico a partir da predição da morbidade e mortalidade (5). Algumas pesquisas têm evidenciado correlação importante da DP no $\mathrm{TC}_{6}$ e variáveis obtidas por testes máximos e de função pulmonar, como o pico máximo de consumo de oxigênio $\left(\mathrm{VO}_{2 \text { máx }}\right)$, ventilação minuto e parâmetros espirométricos $(6,7,8)$. 
Diante de tantos aspectos relevantes para seu uso na prática clínica, tanto do clínico quanto do profissional da fisioterapia, é progressiva sua aplicação também na população pediátrica, seja ela hígida ou na presença de diagnósticos cardiopulmonares, metabólicos, dentre outros $(6,8,9,10,11)$. No entanto, vários são os fatores apontados pela literatura como influentes para um bom desempenho desse teste em crianças, em comparação aos adultos. Dente eles, mudanças relacionadas ao período de crescimento e desenvolvimento infanto-juvenil, como a puberdade e o estirão de crescimento, parecem interferir na DP durante o teste, devido à influência na passada e velocidade da criança. Nesse contexto, é importante identificar os elementos antropométricos que influenciam a DP no $\mathrm{TC}_{6}$ nessa população no sentido de estabelecer as variáveis com potencial poder de confundimento, que dificultam e prejudicam a interpretação dos resultados das pesquisas para que a aplicação desse teste não subestime a capacidade física em doenças crônicas e mascare benefícios de intervenções. Sendo assim, o objetivo desta revisão é analisar publicações referentes ao $\mathrm{TC}_{6}$ realizado em crianças e adolescentes e identificar a influência de parâmetros antropométricos no desempenho do teste.

\section{Materiais e métodos}

Busca na literatura

A busca pelos estudos para compor a revisão foi realizada com pesquisa nas bases de dados eletrônicas SciELO (Scientific Electronic Library Online) e MEDLINE, sem limitação de data, já que publicações sobre o $\mathrm{TC}_{6}$ em crianças e adolescentes são recentes e escassas. As palavras-chave utilizadas foram: six-minute walk test, children, adolescents, anthropometric measures/variables, growth, exercise tolerance. Uma complementação de material foi feita com base nas referências bibliográficas relacionadas ao tema encontradas nos artigos selecionados, sendo elas um abstract (12) e uma tese (13).

\section{Critérios de inclusão dos estudos}

Foram incluídos os estudos envolvendo $\mathrm{TC}_{6} \mathrm{em}$ crianças e/ou adolescentes que apresentaram correlação entre dados antropométricos e variáveis do teste.
Seleção dos estudos

Inseridas as palavras-chave nas duas bases de dados, inicialmente foi feita a leitura dos títulos apresentados e, respeitando-se os critérios de inclusão pré-estabelecidos, foram selecionados 45 estudos potencialmente elegíveis. Os resumos desses trabalhos foram levantados e, após nova triagem, foram identificados 35 estudos, cujos artigos completos foram adquiridos. A partir da análise minuciosa dessas publicações por dois avaliadores independentes, um total de 13 trabalhos foi considerado compatível com o tema e foi incluído nessa revisão. Não foram selecionados os estudos que utilizam o $\mathrm{TC}_{6}$ como parâmetro de avaliação de diferentes intervenções ou como ferramenta de caracterização da amostra, que não apresentam dados sobre a relação entre as variáveis do teste e a antropometria da população investigada.

\section{Resultados}

Já está muito bem estabelecido na literatura o uso do $\mathrm{TC}_{6}$ na população adulta com estudos envolvendo equações de predições com valores de normalidade, fatores influenciadores da DP, em variadas situações clínicas, sendo um instrumento de grande valor em pesquisa clínica para a mensuração da capacidade funcional. A proposta de diretrizes da American Thoracic Society de 2002 guia, orienta e respalda assuntos relacionados ao $\mathrm{TC}_{6}$ na população adulta e, até o momento, tem servido como base técnica também para população pediátrica. No entanto, pesquisadores discutem a aplicabilidade em crianças, a dificuldade de padronização do teste e a escassez de valores e equações de referência para esse grupo. Apesar dessa realidade, o $\mathrm{TC}_{6}$ é usado rotineiramente no manejo do paciente pediátrico e é crescente o número de pesquisas envolvendo sua utilização.

Na corrente revisão, foram identificados estudos com características peculiares. 0 material elencado aborda dados do $\mathrm{TC}_{6}$ e sua aplicação em diferentes situações: análise de sua reprodutibilidade e confiabilidade; seus valores de referência na população infantil saudável e doente; sua indicação em diferentes doenças como parâmetro de efeito de programas de prevenção e reabilitação; sua correlação com teste cardiopulmonar máximo e com dados antropométricos, sendo este último o foco principal da revisão.

O Quadro 1 apresenta os estudos de correlação entre variáveis antropométricas e a $\mathrm{DP}$ pelo $\mathrm{TC}_{6} \mathrm{em}$ crianças e adolescentes. 
Quadro 1 - Relação dos 13 estudos selecionados para a revisão

(Continua)

\begin{tabular}{|c|c|c|c|c|c|}
\hline Autor & $\begin{array}{l}\text { População de } \\
\text { estudo }\end{array}$ & $\begin{array}{l}\text { Tipo de estudo/ } \\
\text { seleção da amostra }\end{array}$ & $\begin{array}{l}\text { Idade } \\
\text { (anos) }\end{array}$ & $\begin{array}{l}\text { Variáveis } \\
\text { antropométricas }\end{array}$ & $\begin{array}{l}\text { Correlação com a } \\
\text { DP no TC } 6\end{array}$ \\
\hline Morinder et al. (8) & $\begin{array}{l}250 \text { crianças e } \\
\text { adolescentes } \\
\text { obesas }\end{array}$ & $\begin{array}{l}\text { teste e re-teste/ } \\
\text { pacientes } \\
\text { ambulatoriais }\end{array}$ & $\begin{array}{l}\text { 8-16 } \\
\text { média de } 13,3\end{array}$ & $\begin{array}{l}\text { - idade; } \\
\text { - altura; } \\
\text { - IMC. }\end{array}$ & $\begin{array}{l}\text { idade }(+) \\
\text { altura }(+) \\
\text { IMC }(-)\end{array}$ \\
\hline Takken et al. (20) & $\begin{array}{l}20 \text { crianças e } \\
\text { adolescentes no } \\
\text { estágio final de } \\
\text { doença renal }\end{array}$ & $\begin{array}{l}\text { Transversal/ } \\
\text { pacientes } \\
\text { em diálise ou } \\
\text { hemodiálise por } \\
\text { mais de } 3 \text { meses }\end{array}$ & $14,1 \pm 3,7$ & $\begin{array}{l}\text { - peso; } \\
\text { - altura; } \\
\text { - IMC; } \\
\text { - \% massa gorda; } \\
\text { - força muscular } \\
\text { de membros } \\
\text { inferiores. }\end{array}$ & altura $(+)$ \\
\hline Hassan et al. (12) & $\begin{array}{l}47 \text { crianças } \\
\text { hemofílicas (1) } \\
44 \text { com AlJ (2) } \\
22 \text { com Espinha } \\
\text { Bífida (3) }\end{array}$ & $\begin{array}{l}\text { Transversal/ } \\
\text { pacientes seguidos } \\
\text { em clinicas } \\
\text { e hospitais } \\
\text { universitários }\end{array}$ & $\begin{array}{r}12,5 \pm 2,9 \\
9,3 \pm 2,9 \\
10,3 \pm 3,1\end{array}$ & $\begin{array}{l}\text { - idade; } \\
\text { - altura; } \\
\text { - peso. }\end{array}$ & $\begin{array}{l}\text { 1- idade, peso e } \\
\text { altura }(+) \\
2 \text { - altura }(+) \\
3 \text { - sem correlação }\end{array}$ \\
\hline Calders et al. (21) & $\begin{array}{l}64 \text { crianças e } \\
\text { adolescentes } \\
\text { obesos }\end{array}$ & $\begin{array}{l}\text { Prospectivo/ } \\
\text { obesos antes e } \\
\text { após tratamento } \\
\text { multidisciplinar }\end{array}$ & $\begin{array}{l}\text { 10-18 } \\
\text { média de } 14,7\end{array}$ & $\begin{array}{l}\text { - altura; } \\
\text { - peso; } \\
\text { - IMC; } \\
\text { - \% massa gorda; } \\
\text { - \% massa magra; } \\
\text { - circunferência } \\
\text { abdominal. }\end{array}$ & $\begin{array}{l}\text { altura }(+) \\
\text { peso }(-) \\
\text { IMC }(-) \\
\% \text { massa gorda }(-) \\
\% \text { massa magra }(+) \\
\text { Circunferência } \\
\text { abdominal }(-)\end{array}$ \\
\hline Li et al. (15) & $\begin{array}{l}1445 \text { crianças } \\
\text { e adolescentes } \\
\text { saudáveis }\end{array}$ & $\begin{array}{l}\text { Transversal/ } \\
\text { recrutados } \\
\text { randomicamente de } \\
\text { escolas }\end{array}$ & $12 \pm 2,6$ & $\begin{array}{l}\text { - idade; } \\
\text { - peso; } \\
\text { - altura; } \\
\text { - IMC; } \\
\text { - sexo. }\end{array}$ & $\begin{array}{l}\text { idade }(+) \\
\text { peso }(+) \text { em } \\
\text { meninos } \\
\text { altura }(+) \\
\text { IMC }(-) \text { em } \\
\text { meninas } \\
\text { sexo masculino } \\
(+)\end{array}$ \\
\hline Lammers et al. (17) & $\begin{array}{l}328 \text { crianças } \\
\text { saudáveis }\end{array}$ & $\begin{array}{l}\text { Transversal/ } \\
\text { recrutadas } \\
\text { randomicamente de } \\
\text { escolas }\end{array}$ & $4-11$ & $\begin{array}{l}\text { - idade; } \\
\text { - sexo; } \\
\text { - altura; } \\
\text { - IMC; } \\
\text { - peso. }\end{array}$ & $\begin{array}{l}\text { idade }(+) \\
\text { peso }(+) \\
\text { altura }(+) \\
\text { sexo masculino }(+)\end{array}$ \\
\hline Geiger et al. (16) & $\begin{array}{l}528 \text { crianças e } \\
\text { adolescentes } \\
\text { saudáveis }\end{array}$ & $\begin{array}{l}\text { Transversal/ } \\
\text { voluntários } \\
\text { caucasianos nas } \\
\text { escolas }\end{array}$ & $3-11$ & $\begin{array}{l}\text { - idade; } \\
\text { - peso; } \\
\text { - altura; } \\
\text { - IMC; } \\
\text { - comprimento de } \\
\text { MMII. }\end{array}$ & $\begin{array}{l}\text { idade }(+) \\
\text { altura }(+)\end{array}$ \\
\hline
\end{tabular}


Quadro 1 - Relação dos 13 estudos selecionados para a revisão

(Continua)

\begin{tabular}{|c|c|c|c|c|c|}
\hline Autor & $\begin{array}{l}\text { População de } \\
\text { estudo }\end{array}$ & $\begin{array}{l}\text { Tipo de estudo/ } \\
\text { seleção da amostra }\end{array}$ & $\begin{array}{l}\text { Idade } \\
\text { (anos) }\end{array}$ & $\begin{array}{l}\text { Variáveis } \\
\text { antropométricas }\end{array}$ & $\begin{array}{l}\text { Correlação com a } \\
\text { DP no TC } 6\end{array}$ \\
\hline Oliveira (13) & $\begin{array}{l}102 \text { crianças e } \\
\text { adolescentes } \\
\text { saudáveis }\end{array}$ & $\begin{array}{l}\text { Transversal/ } \\
\text { selecionados } \\
\text { randomicamente de } \\
\text { uma escola }\end{array}$ & $12,9 \pm 3$ & $\begin{array}{l}\text { - peso; } \\
\text { - altura; } \\
\text { - idade; } \\
\text { - gênero; } \\
\text { - IMC; } \\
\text { - comprimento de } \\
\text { MMIl; } \\
\text { - circunferência de } \\
\text { coxa; } \\
\text { - \% massa magra; } \\
\text { - \% massa gorda; } \\
\text { - força muscular } \\
\quad \text { respiratória. }\end{array}$ & $\begin{array}{l}\text { peso }(+) \\
\text { altura }(+) \\
\text { idade }(+) \\
\text { comprimento de } \\
\text { MMIl }(+) \\
\text { circunferência de } \\
\text { coxa }(+)\end{array}$ \\
\hline $\begin{array}{l}\text { Li et al. } \\
\text { (14) }\end{array}$ & $\begin{array}{l}78 \text { crianças } \\
\text { chinesas saudáveis }\end{array}$ & $\begin{array}{l}\text { Teste e re-teste/ } \\
\text { escolares } \\
\text { recrutados } \\
\text { randomicamente }\end{array}$ & $14,2 \pm 1,1$ & $\begin{array}{l}\text { - altura; } \\
\text { - idade; } \\
\text { - peso; } \\
\text { - IMC. }\end{array}$ & altura (+) \\
\hline Cunha et al. (9) & $\begin{array}{l}16 \text { crianças e } \\
\text { adolescentes com } \\
\text { Fibrose Cística }\end{array}$ & $\begin{array}{l}\text { Transversal/ } \\
\text { escolares e } \\
\text { adolescentes } \\
\text { clinicamente } \\
\text { estáveis }\end{array}$ & $11 \pm 1,9$ & $\begin{array}{l}\text { - idade; } \\
\text { - peso; } \\
\text { - altura; } \\
\text { - IMC. }\end{array}$ & sem correlação \\
\hline Basaran et al. (19) & $\begin{array}{l}62 \text { crianças } \\
\text { com asma de } \\
\text { sintomatologia de } \\
\text { média a moderada }\end{array}$ & $\begin{array}{l}\text { Prospectivo/ } \\
\text { pacientes } \\
\text { ambulatoriais } \\
\text { randomizados } \\
\text { em dois grupos: } \\
\text { controle e exercício }\end{array}$ & $10,4 \pm 2,1$ & $\begin{array}{l}\text { - idade; } \\
\text { - sexo; } \\
\text { - altura; } \\
\text { - IMC. }\end{array}$ & sexo masculino (+) \\
\hline Moalla et al. (11) & $\begin{array}{l}17 \text { crianças e } \\
\text { adolescentes } \\
\text { com cardiopatia } \\
\text { congênita }\end{array}$ & $\begin{array}{l}\text { Teste e re-teste/ } \\
\text { Cardiopatas classe } \\
\text { II ou III }\end{array}$ & $13,5 \pm 0,5$ & $\begin{array}{l}\text { - idade; } \\
\text { - peso; } \\
\text { - altura; } \\
\text { - IMC. }\end{array}$ & sem correlação \\
\hline Nixon et al. (6) & $\begin{array}{l}17 \text { crianças e } \\
\text { adolescentes } \\
\text { gravemente } \\
\text { doentes }\end{array}$ & $\begin{array}{l}\text { Teste e re-teste/ } \\
\text { pacientes } \\
\text { hospitalizados } \\
\text { com indicação de } \\
\text { transplante coração } \\
\text { e/ou pulmão }\end{array}$ & $14,8 \pm 2,6$ & - altura. & sem correlação \\
\hline
\end{tabular}

Legenda: $\mathrm{TC}_{6}=$ teste de caminhada de seis minutos; IMC $=$ índice de massa corporal; $\mathrm{AlJ}=$ artrite idiopática juvenil; $\mathrm{FC}=$ fibrose cística; MMII = membros inferiores; $(+)=$ correlação positiva com a DP pelo $\mathrm{TC}_{6} ;(-)=$ correlação negativa com a DP pelo $\mathrm{TC}_{6}$.

Fonte: Dados da pesquisa. 


\section{Discussão}

Valores de referência do $\mathrm{TC}_{6}$ em pediatria e relação com parâmetros antropométricos

Dos 13 artigos incluídos nessa revisão, quatro estudos sugeriram valores de referência no $\mathrm{TC}_{6}$ para crianças e analisaram a relação entre a DP e parâmetros antropométricos. A forte influência positiva da altura na distância foi um dos resultados de alguns estudos $(14,15)$. Os autores (14) investigaram a validade e confiabilidade do $\mathrm{TC}_{6}$ e estabeleceram valores de referência após analisar 78 crianças e adolescentes chineses saudáveis entre 7 e 16 anos; também verificaram correlação da distância com sexo, idade e dados antropométricos de peso e índice de massa corporal (IMC), porém sem significância. 0 estudo posterior (15) avaliou 1.445 crianças e adolescentes e estabeleceu curvas de normalidade para a população chinesa; observou correlação da DP pelo $\mathrm{TC}_{6}$ com idade, sexo, peso (em meninos), IMC (em meninas), sendo a altura a variável de maior influência.

A relação entre DP e sexo, idade e altura de crianças foi novamente constatada por Geiger et al. (16), que propuseram equações para valores de referência envolvendo essas variáveis, consideradas responsáveis pela variabilidade na $\mathrm{DP}$ durante o $\mathrm{TC}_{6}$ modificado aplicado em 528 crianças e adolescentes saudáveis de 3 a 11 anos de idade; o teste foi realizado com o incremento de uma roda adaptada para ser conduzida à frente do corpo da criança. Na mesma linha, Lammers (17) avaliou 328 crianças saudáveis, entre 4 e 11 anos, e também observou correlação da DP com peso, altura e idade, sendo esta última relacionada ao aumento progressivo da trajetória. Diferente de Geiger et al. (16), não houve diferença entre a performance de meninos e meninas.

A frequente intervenção do gênero, idade, peso, altura e IMC no resultado do $\mathrm{TC}_{6}$ motivou Oliveira (13) a investigar a influência desses, e de outros fatores como superfície corporal, comprimento de membros inferiores, força muscular respiratória, circunferência de coxa, metabolismo basal e percentual de massa magra e gorda. Para essa pesquisa, sua amostra foi composta de 102 indivíduos e houve correlação positiva da distância com idade, peso, altura, superfície corporal, comprimento de membros inferiores, circunferência de coxa e metabolismo basal. Com base nesses resultados, esse autor também elaborou uma equação de referência para predição da DP nessa população, de acordo com o gênero, e considerando a idade, peso e altura, variáveis que contribuíram com a maior porcentagem de variabilidade no resultado do $\mathrm{TC}_{6}$.

Fica evidente que, grande parte dos estudos aqui selecionados relaciona a DP no $\mathrm{TC}_{6}$ com a idade e o sexo. Com relação à idade, estudos atribuem uma maior velocidade do $\mathrm{TC}_{6}$ com o aumento da idade $(8,12,13,15-17)$, e naqueles com maior IMC, em decorrência das mudanças fisiológicas que ocorrem durante o período de crescimento e desenvolvimento infanto-juvenil. Por esse motivo, a maioria deles analisa uma faixa etária restrita, com o intuito de controlar todas as variações biotípicas. Esse fato já difere dos adultos, que apresentam um comportamento mais previsível durante o teste $(17,18)$.

Sabe-se que existem inúmeras variações físicas entre adultos, de um lado, e adolescentes e crianças, de outro, e que essas diferenças são muito mais complexas que as medidas antropométricas. A fisiologia infantil muda constantemente porque seus sistemas estão em processo de crescimento, desenvolvimento e maturação, sofrendo influências relacionadas à genética e aos fatores extrínsecos: nível de atividade física, composição corporal, estado nutricional, condição socioeconômica, cultura, gênero, etnia, clima e localização geográfica. Dessa forma, a população pediátrica tem um padrão singular de mudanças em seu organismo, inclusive em termos comportamentais, que afeta seu desempenho físico e pode alterar suas respostas fisiológicas durante a prática do exercício físico (13) ou de testes físicos, como é o caso do $\mathrm{TC}_{6}$. Pesquisas futuras devem considerar todos esses aspectos no intuito de utilizar o teste da forma mais eficaz possível e de conhecer cada vez mais o impacto de diferentes fatores na performance do teste, sejam eles fatores físicos, hormonais ou sociais.

Como exposto, a influência das variáveis como altura $(8,12-17,19-21)$, peso $(12,13,15,17,21)$ e IMC $(8,15,21)$, bem como da porcentagem de massa gorda e magra (21) tem sido frequentemente investigada nos trabalhos relacionados à performance do teste em crianças, sendo a estatura um dos elementos de maior impacto. 0 comprimento de membros inferiores (13), a circunferência abdominal (21) e de coxas (13) também são variáveis exploradas nas pesquisas e apresentam relação com o desempenho na DP, assim como o gênero - os meninos apresentam melhores resultados $(15,17,19)$. 
Em relação ao estudo da reprodutibilidade do $\mathrm{TC}_{6}$ em diferentes populações, foram identificadas duas pesquisas bem-sucedidas que fizeram essa abordagem em situações especiais. Cunha et al. (9) constatou a reprodutibilidade do $\mathrm{TC}_{6}$ em 16 crianças e adolescentes com fibrose cística e Morinder et al. (8) fez a mesma constatação em crianças e adolescentes obesos.

Existe grande relevância no estudo da confiabilidade e reprodutibilidade do teste, uma vez que essa constatação reforça sua aplicação como um instrumento de avaliação funcional seguro, principalmente em situações de manejo de enfermidades e monitorização clínica.

$\mathrm{TC}_{6}$ e relação com parâmetros antropométricos de crianças doentes

Foram identificados cinco estudos que aplicaram o $\mathrm{TC}_{6}$ em populações de crianças doentes e, diferente dos estudos com crianças saudáveis anteriormente mencionados, a maioria deles não identificou influência de parâmetros antropométricos no resultado do teste.

Nessa perspectiva, a correlação entre os parâmetros de IMC, variáveis espirométricas, escores de gravidade, pressão inspiratória máxima, pressão expiratória máxima, trabalho realizado pelo $\mathrm{TC}_{6}$ (variável correspondente à DP pelo teste multiplicado pelo peso corporal) e a performance do teste foi estudada em crianças e adolescentes com fibrose cística, sendo interferentes apenas os dois últimos dados (9).

Um estudo com crianças com cardiopatia congênita não evidenciou relação da altura, idade, peso e IMC na DP no $\mathrm{TC}_{6}$ (11). A mesma ausência de relação entre essas variáveis antropométricas e o teste foi constatada em crianças com asma, de sintomatologia média a moderada (19), em crianças graves com indicação de transplante pulmonar, cardíaco ou ambos (6) e doentes renais pediátricos graves (20). Estes últimos também não apresentaram influência da porcentagem de massa gorda e força muscular de membros inferiores na DP no $\mathrm{TC}_{6}$. Houve relação entre o desempenho no $\mathrm{TC}_{6}$, a altura e a quantidade de hematócrito e, diante desse fato, os autores relataram ser esse teste um instrumento útil para monitorizar o estado clínico e funcional desse tipo de paciente (21).

A maioria dos trabalhos envolvendo crianças saudáveis havia identificado influência da altura na performance do $\mathrm{TC}_{6}$ (14-17), mas, diferentemente, esse parâmetro não teve relação com as pesquisas supracitadas envolvendo amostra de doentes $(6,11$, $19,20)$. Apesar desse frequente comportamento, a altura foi a variável antropométrica de maior influência no teste de crianças obesas e em doenças com algum caráter degenerativo $(8,12,21)$.

Em crianças e adolescentes obesos, variáveis como idade, altura e IMC influenciaram no resultado do $\mathrm{TC}_{6}$ $(8,21)$, além do peso, circunferência abdominal e porcentagem de massas gorda e magra (21). Nesse perfil de crianças, a capacidade funcional parece ser inferior àquelas com peso ideal, pois elas caminham menos em comparação a crianças saudáveis de peso ideal (8).

No segundo caso, em doenças degenerativas, pacientes com três tipos de enfermidades crônicas hemofilia, artrite idiopática juvenil e espinha bífida - foram investigados em relação aos parâmetros antropométricos que interferiam mais na $\mathrm{DP}$ do $\mathrm{TC}_{6}$; com exceção da altura, os autores verificaram respostas diferentes para cada uma das doenças (12).

Considerando os estudos referenciados, torna-se relevante pontuar que muitos são os fatores que afetam a DP no $\mathrm{TC}_{6}$, tanto de forma negativa (estatura baixa; membros inferiores curtos; idade avançada; peso corporal elevado; sexo feminino; cognição prejudicada; corredor para caminhada menor, sendo necessário maior número de voltas; doenças crônicas de ordem respiratória, cardiovascular e ortopédica) como positivamente (estatura alta; sexo masculino; grande motivação; treinamento realizado antes do teste; determinadas medicações e suplemento de oxigênio) (3). Dependendo da doença estudada, características clínicas, presença de complicações e o próprio quadro do paciente no momento da realização do exame podem afetar, significativamente, o desempenho no $\mathrm{TC}_{6}$.

É de grande valor em pesquisas clínicas nessa área correlacionar adequadamente as variáveis antropométricas com o resultado do $\mathrm{TC}_{6}$ a fim de evitar interpretações errôneas. Comumente atribuem-se pequenas distâncias percorridas a doenças crônicas com baixa capacidade funcional, sem considerar que esses pacientes geralmente apresentam baixa estatura e grande dificuldade no ganho de peso comprometido.

\section{$\mathrm{TC}_{6}$ e relação com testes máximos}

$0 \mathrm{TC}_{6}$ também tem sido utilizado para avaliação de tolerância ao exercício e endurance, sendo considerado 
um teste funcional válido e confiável (14). Com essa finalidade, o trabalho de Moalla et al. (11) correlacionou valores do $\mathrm{TC}_{6}$ com variáveis de teste cardiopulmonar máximo comparando a tolerância ao exercício e resposta cardiorrespiratória de crianças com cardiopatia congênita e um grupo controle de crianças saudáveis, e observou relação significante entre valores de $\mathrm{VO}_{2 \text { máx }}$ e DP. Nixon et al. (6) já havia avaliado a eficácia do $\mathrm{TC}_{6}$ como um método alternativo para avaliar capacidade funcional de indivíduos com tolerância física muito limitada, em estudo que analisou 17 crianças gravemente doentes, candidatas a transplante pulmonar, cardíaco ou ambos. Em sua pesquisa, houve correlação significante entre o $\mathrm{VO}_{2 \text { máx }}$ medido através do teste cardiopulmonar máximo e a $\mathrm{DP}$ no $\mathrm{TC}_{6}$.

Em saudáveis, Li et al. (14) observaram relação com o $\mathrm{VO}_{2 \text { máx }}$ e o volume expiratório forçado no primeiro segundo $\left(\mathrm{VEF}_{1}\right)$ após analisar 78 crianças e adolescentes chinesas saudáveis. 0 teste também foi considerado um bom instrumento para avaliação da tolerância ao exercício em obesos, apesar de ter sido verificada baixa correlação entre $\mathrm{DP}$ e $\mathrm{VO}_{2 \text { máx }}$ no estudo de Morinder et al. (8).

\section{$\mathrm{TC}_{6}$ como resposta a intervenções e terapêuticas}

A indicação do $\mathrm{TC}_{6}$ como um instrumento de monitorização e avaliação a diferentes tratamentos tem sido utilizado rotineiramente no manejo de diferentes situações clinicas $(19,21,22)$, principalmente nas doenças cardiorrespiratórias crônicas.

Nessa linha, crianças com asma média a moderada foram analisadas em pesquisa que utilizou o $\mathrm{TC}_{6}$ para avaliar a capacidade de exercício após um programa de exercícios respiratórios domiciliares com duração de oito semanas. $0 \mathrm{TC}_{6}$ mostrou-se sensível em reproduzir os benefícios dessa intervenção (19). Moalla et al. (11) compararam a tolerância ao exercício e resposta cardiorrespiratória de crianças com cardiopatia congênita após um programa de treinamento físico por 12 semanas. Os cardiopatas treinados apresentaram melhora na $\mathrm{DP}$ no $\mathrm{TC}_{6}$, evidenciando ser este um teste sensível para avaliar capacidade funcional nessa população. Em crianças e adolescentes obesos, a repercussão de um tratamento multidisciplinar na relação entre preditores e o resultado do $\mathrm{TC}_{6}$ também foi foco de avaliação (21).

Nesse contexto, é importante relatar que os estudos epidemiológicos envolvendo crescimento e aptidão física (22) e pesquisas sobre avaliação do efeito de intervenções em longo prazo podem constatar resultados mascarados, e até "falso positivos", quando não são considerados os efeitos fisiológicos que ocorrem na fase de maturação física infanto-juvenil. Crianças e adolescentes sofrem influência de transformações fisiológicas e anatômicas decorrentes das descargas hormonais, que têm sua secreção aumentada nesse período. Com o rápido crescimento somático, e consequente aumento da massa muscular que está intimamente ligado ao consumo de oxigênio, há o aumento de órgãos como os pulmões e o coração. Ambos têm influência direta na melhora da resistência cardiorrespiratória, em virtude do aumento na captação e utilização do oxigênio. Estudos envolvendo grupos nessa fase de desenvolvimento podem detectar equivocadamente benefícios decorrentes de intervenções, podendo estar relacionados exclusivamente a mudanças fisiológicas naturais da fase em que a criança se encontra.

Diante do exposto até aqui, é fundamental discutir a característica dos estudos incluídos nesta revisão. Tais estudos transportam o modelo do teste do adulto para população pediátrica, apresentam amostras pequenas e métodos divergentes de aplicação do $\mathrm{TC}_{6}$ referentes a diferenças no tamanho do corredor e no comando verbal de encorajamento, ou até ao tempo de intervalo entre dois testes e o efeito do treinamento/aprendizagem.

Questões inerentes à padronização do teste são de extrema relevância (1-3), são essenciais para garantir a confiabilidade dos resultados obtidos com sua aplicação. Sabe-se que o melhor desempenho no $\mathrm{TC}_{6}$ é dependente de aprendizado, motivação e esforço e, para que esses elementos sejam estimulados, principalmente na faixa etária pediátrica, deve haver controle de todas as normas técnicas pertinentes à sua execução. Além das variáveis antropométricas e a presença de enfermidades, como já apresentado, todos esses fatores teórico-práticos interferem diretamente no resultado do teste.

Sendo assim, são necessários novos estudos, específicos e bem delineados, que avaliem a correlação entre $\mathrm{TC}_{6}$ e variáveis antropométricas, além de outros fatores que podem influenciar no teste. Esses dados servirão de subsídio para a elaboração de equações de referência e valores de normalidade mais fidedignos, além de embasamento teórico-científico para organização de diretrizes do $\mathrm{TC}_{6}$ em crianças e adolescentes. 


\section{Considerações finais}

É indiscutível a importância do $\mathrm{TC}_{6}$ no manejo de crianças e adolescentes, sejam eles saudáveis ou doentes. Mas fica evidente que se deve considerar a forte influência entre variáveis antropométricas e o desempenho no teste, já que são potenciais fatores de confundimento. Sugere-se que trabalhos que utilizem o $\mathrm{TC}_{6}$ sejam cuidadosos na análise dos resultados e considerem a influência da antropometria, especialmente altura, peso e IMC, bem como da porcentagem de massa gorda e magra, comprimento de membros inferiores, força muscular respiratória e de membros inferiores. Além disso, de acordo com a idade, devem-se considerar os aspectos inerentes ao crescimento e desenvolvimento infantil. Esclarecer essas associações contribui para evitar resultados funcionais de pouca acurácia, para o pesquisador e clínico, e o comprometimento do desfecho de estudos e terapêuticas.

\section{Referências}

1. Du H, Newton PJ, Salamonson Y, Carrieri-Kohlman VL, Davidson PM. A review of the six-minute walk test: Its implication as a self-administered assessment tool. Eur J Cardiov Nursing. 2009;8(1):2-8. doi:10.1016/j. ejcnurse.2008.07.001.

2. Enright PL. The six-minute walk test. Respir Care. 2003;48(8):783-5. PMid:12890299.

3. American Thoracic Society. ATS Statement: Guidelines for the six-minute walk test. Am J Respir Crit Care Med. 2002;166(1):111-17. PMid:12091180.

4. Noonan V, Dean E. Submaximal exercise testing: clinical application and interpretation. Phys Ther. 2000;80(8):78-807.

5. Solway S, Brooks D, Lacasse Y, Thomas S. A qualitative systemic overview of the measurement properties of functional walk tests used in the cardiorespiratory domain. Chest. 2001;119(1):256-270. doi:10.1378/ chest.119.1.256.

6. Nixon PA, Joswiak ML, Fricker FJ. A six-minute walk test for assessing exercise tolerance in severely ill children. J Pediatr. 1996;129(3):362-6. doi:10.1016/ S0022-3476(96)70067-7.
7. Cahalin L, Pappagianopoulos P, Prevost S, Wain J, Ginns L. The relationship of the 6-min walk test to maximal oxygen consumption in transplant patients with end-stage lung disease. Chest. 1995;108(2):452-9. doi:10.1378/chest.108.2.452.

8. Morinder G, Mattsson E, Sollander C, Marcus C, Larsson UE. Six-minute walk test in obese children and adolescents: reprodutibility and validity. Physiother Res Int. 2009; 14(2):91-104. doi:10.1002/pri.428.

9. Cunha MT, Rozov T, de Oliveira RC, Jardim JR. Six-minute walk test in children and adolescents with cystic fibrosis. Pediatr Pulmonol. 2006;41(7):618-22. doi:10.1002/ppul.20308.

10. Mattiello R, Sarria EE, Stein R, Fischer GB, Mocelin HT, Barreto SSM, et al. Functional capacity assessment during exercise in children and adolescents with post-infectious bronchiolitis obliterans. J Pediatr (Rio J). 2008; 84(4):337-43. doi:10.2223/JPED.1807.

11. Moalla W, Gauthier R, Maingourd Y, Ahmaidi S. Six-minute walking test to assess exercise tolerance and cardiorespiratory responses during training program in children with congenital heart disease. Int J Sports Med. 2005;26(9):756-62. doi:10.1055/s-2004-830558.

12. Hassan J, van der Net J, Helders PJ, Prakken BJ, Takken T. Six-minute walking test in children with chronic conditions. Br J Sports Med. 2010;44(4):270-4. doi:10.1136/bjsm.2008.048512.

13. Oliveira AC. Equação de referência para o teste de caminhada de seis minutos em crianças e adolescentes saudáveis. [dissertação]. Belo Horizonte (MG): Universidade Federal de Minas Gerais; 2007.

14. Li AM, Yin J, Yu CC, Tsang T, So HK, Wong E, et al. The six-minute walk test in healthy children: reliability and validity. Eur Respir J. 2005;25(6):1057-60. doi:1 0.1183/09031936.05.00134904.

15. Li AM, Yin J, Au JT, So HK, Tsang T, Wong E, et al. Standard reference for the 6-minute walk test in healthy children aged 7 to 16 years. Am J Respir Crit Care Med. 2007;176(2):174-80. doi:10.1164/rccm. 200607-8830C.

16. Geiger R, Strasak A, Treml B, Gasser K, Kleinsasser A, Fischer V, et al. Six-minute walk test in children and adolescents. J Pediatr. 2007;150(4):395-9. doi: 10.1016/j.jpeds.2006.12.052. 
17. Lammers AE, Hislop AA, Flynn Y, Haworth SG. The 6-minute walk test: normal values for children of 4-11 years of age. Arch Dis Child. 2008;93(6):464-8. doi:10.1136/adc.2007.123653.

18. Pires SR, Oliveira AC, Parreira VF, Brito RR. Teste de caminhada de seis minutos em diferentes faixas etárias e índices de massa corporal. Rev Bras Fisioter. 2007;11(2): 147-51. doi:10.1590/ S1413-35552007000200010.

19. Basaran S, Guler-Uysal F, Ergen N, Seydaoglu G, Bingol-Karakoç G, altintas DU. Effects of physical exercise on quality of life, exercise capacity and pulmonary function in children of asthma. J Rehabil Med. 2006;38(2):1305. doi:10.1080/16501970500476142.

20. Takken T, Engelbert R, van Bergen M, Grothoff J, Nauta J, van Hoeck K, et al. Six-minute walking test in children with ESRD: discrimination validity and construct validity. Pediatr Nephrol. 2009;24(11):2217-23. doi:10.1007/s00467-009-1259-x.
21. Calders P, Deforche B, Verschelde S, Bouckaert J, Chevalier F, Bassle E, et al. Predictors of 6-minute walk test and 12-minute walk test in obese children and adolescents. Eur J Pediatr. 2008;167(5):563-8. doi:10.1007/s00431-007-0553-5.

22. Bergmann GG, Araújo MLB, Garlipp DC, Lorenzi TDC, Gaya A. alteração anual no crescimento e na aptidão física relacionada à saúde de escolares. Rev Bras Cineantropom Desempenho Hum. 2005;7(2):55-61.

Recebido: $11 / 02 / 2012$

Received: 02/11/2012

Aprovado: 29/09/2012

Approved: 09/29/2012 